\title{
Impact of Static Magnetic Field Exposure on the Development and Transcriptome of Medaka Fish Embryos
}

\author{
Weinong Sun ${ }^{1 *}$, Sai-Wing Leung ${ }^{2}$ and Yuen Chong Kong ${ }^{3}$ \\ ${ }^{1}$ Department of Electrical Engineering, City University of Hong Kong, 83 Tat Chee Avenue, China \\ ${ }^{2}$ EMC Consortium Limited, Hong Kong, 2101, 21/F Gala Place, 56 Dundas Street, China \\ ${ }^{3}$ Department of Chemistry, City University of Hong Kong, 83 Tat Chee Avenue, China
}

*Corresponding author: Weinong Sun, Department of Electrical Engineering, City University of Hong Kong, 83 Tat Chee Avenue, Hong Kong, China

\section{ARTICLE INFO}

Received: 幽 October 23, 2020

Published: 慧 November 11, 2020

Citation: Weinong Sun, Sai-Wing Leung, Yuen Chong Kong. Impact of Static Magnetic Field Exposure on the Development and Transcriptome of Medaka Fish Embryos. Biomed J Sci \& Tech Res 31(5)-2020. BJSTR. MS.ID.005158.

Keywords: Static magnetic field; Medaka fish; In vivo; Embryo growth; Transcriptome

\begin{abstract}
The impact of static magnetic field (SMF) exposure on the medaka fish has been investigated recently elucidating the interaction mechanisms of SMF exposure to human cells. A recent study of the in-vivo medaka fish embryo experiment has suggested that there was no obvious impact on the developmental progress of the embryos growth rate under prolonged SMF exposure; however, an increase in percentage of abnormal fish embryos was observed. This paper focuses on transcriptomic analysis of SMFaffected medaka fish embryos at various stages of development following prolonged SMF exposure. This paper verified that under prolonged exposure of SMF there was no significant impact on the embryo growth rate based on the examination of a total of 150 fish samples. However, there was an observable difference in the number of abnormal embryos between the treated and control groups at developmental stage 22 to stage 39 of the embryos. Transcriptomic analysis of biological processes by RNA sequencing were hence carried out; the outcomes of the functional annotation of the KEGG pathways revealed differential expression of biological processes related to ribosomal diseases, and to digestion and absorption of fat in the treated groups when compared to the control groups with p-values of less than 0.05 .
\end{abstract}

\section{Introduction}

There are unintentional static magnetic fields (SMFs) with magnitudes hundreds of times larger than that of the earth's magnetic field existing in our recent environments; and is causing potentially serious public health and safety concerns. Electrified railway or transportation systems, e-vehicles and several major technologies involving DC power energy conversion are considered as common unintentional man-made sources that expose humans to prolonged SMF exposures. For example, some models of hybrid electric vehicles are reporting a $1 \mathrm{mT}$ SMF intensity [1]; while US and European transportation systems have recorded SMF levels of 2 $\mathrm{mT}$ in the passenger cabins of trains [2-4]. An average of $10 \mathrm{mT}$ was measured in electrolytic processing plants, a maximum to $50 \mathrm{mT}$ has also been reported at some accessible locations in superconducting systems for DC power energy conversion technology, and up to 100 $\mathrm{mT}$ has been measured in aluminum production plants [5]. Long term SMF exposures have raised public health concerns in recent decades; ICNIRP [6] has been investigating the potential ill effects of SMF exposure by using cell or animal models to assess the possible health risk. There are so far insufficient in-vitro studies of human cells to determine any health effects caused by electromagnetic radiation. In the absence of any conclusive evidence from in-vivo studies, there has been insufficient corroborating evidence linking SMF exposure human health risks.

It is reported that SMF will affect some endpoints of in-vitro cellular changes at a low intensity magnetic field [7] at mT range. When rat lymphocytes were exposed to a SMF intensity of $7 \mathrm{mT}$ in 
the presence of ferrous chloride, the number of damaged cells was significantly increased [8]. When Reina et al. [9] exposed cellular membrane seeds to magnetic fields of 0 to $10 \mathrm{mT}$, it was observed that there were changes in the magnitude of the current density of ions across the cell membrane and in the ionic concentration with an increased dosage of the field intensity. Hirai [10] investigated the gene expression in hippocampal neurons of rats; a brief exposure of only 15 minutes SMF of $100 \mathrm{mT}$ would lead to a transient potent increase of DNA immaturity. Amara [11] investigated the effects of SMF to $128 \mathrm{mT}$ with 1 hour per day for a consecutive 30 days exposure together with anti-oxidative enzymes activity in a male rat brain; the study indicated that the exposure to SMF will induce oxidative stress in the rat's hippocampus and frontal cortex. Sun [12] has adopted medaka fish to investigate the embryo development as a pilot in-vivo study with no observable impact on the embryo development rate under prolonged SMF exposure. However, unusual abnormal growth was observed in some embryo samples of the treated groups. It is the aim of this paper to carry out a second-round experiment on a larger sample size for further evidential data on the observation of the edema growth together with transcriptome analysis by RNA sequencing.

\section{Methodology}

The experiment was carried out with a sample size of 150 embryos, with 75 embryos of the treated groups and with the same number of 75 embryos of the control groups. 15 embryos were placed in each petri dish for the experiment. SMF exposure for the treated groups was adopted to a full hatching period of 19 days. The SMF was setup by two paralleled NdFeB magnets, the petri dishes of the treatment group were placed between the magnets, and the 15 embryos in each dish were placed in the middle of the dish as the exposure region with water droplets extruded from a dropper. The magnetic flux density within the embryo exposure region was between 80-100 mT measured by a GM05 Gaussmeter. Details of the experimental set up is according to the pilot study as previously reported [12]. The setup is illustrated in (Figures 1-3).

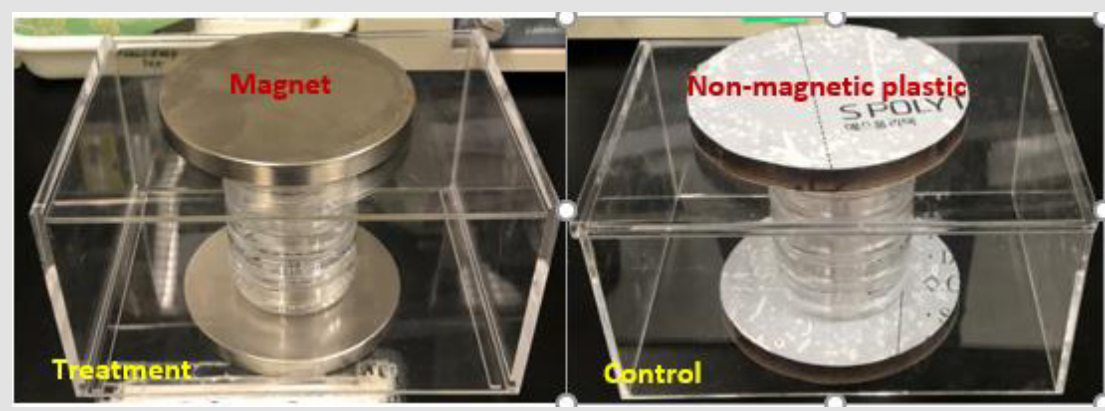

Figure 1: Experimental setup for the exposure of the embryos.

\section{Embryo Developmental Stages}

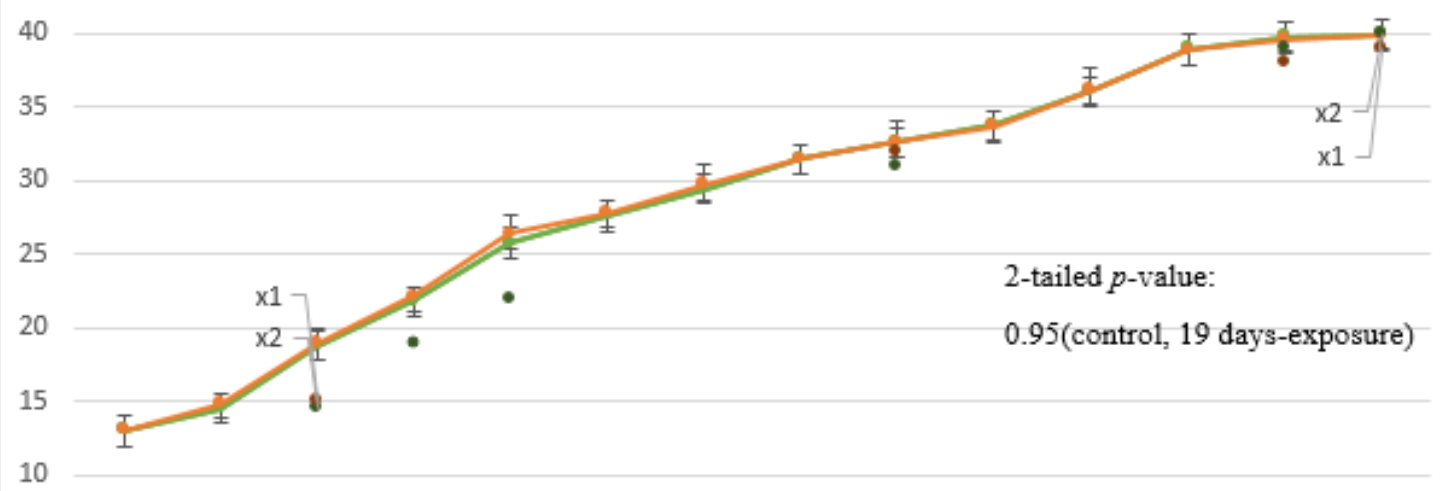

day 1 day 2 day 3 day 4 day 6 day 7 day 8 day 9 day 10 day 11 day 13 day 16 day 17 day 19 —C Control —-Treated - Death •

Figure 2: Embryo developmental stages of both the treated and the control groups. 

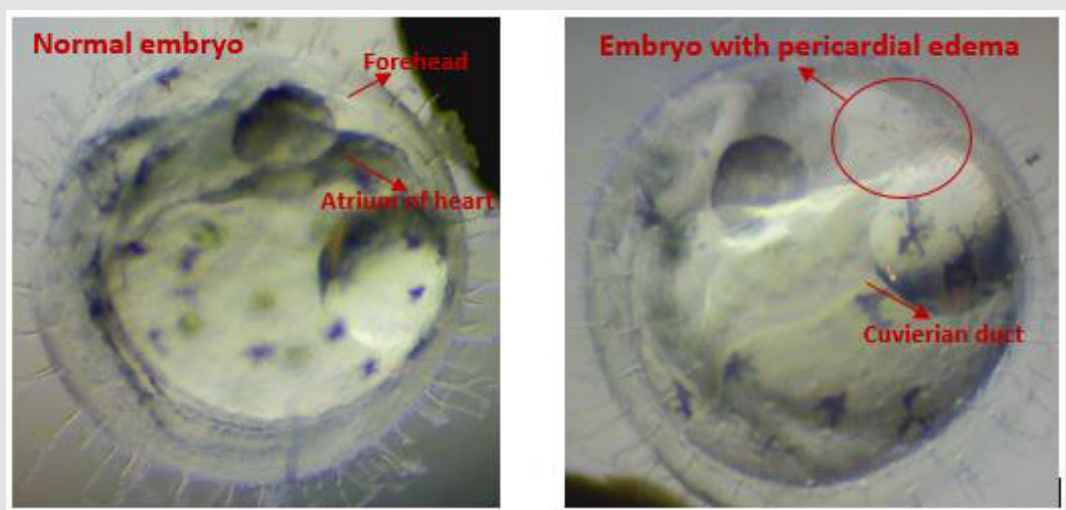

Figure 3: The appearance of the unusual edema growth of the embryo as compared to the normal embryo.

\section{Results}

Figure 2 shows the observed embryo developmental stages in the experiment; no significant impact was observed on the embryo growth rate under prolonged exposure to SMF. Only a marginal difference in growth rate was observed in embryos at developmental stages 22-27; this is consistent with the outcomes observed in the pilot study. The early embryo death in both groups was mainly caused by eggshell rupture likely caused by embryo mishandling during the experiment. Although no significant difference in the embryo growth rate between the treated and the control groups was observed; abnormal embryo growth was observed between stages
22-39: abnormal growth was approximately $8 \%$ of embryos in the treated groups and $1.3 \%$ in the control groups. The embryos with abnormal growth in the treated and control groups are illustrated and listed in (Tables 1 \& 2), respectively. Abnormal edema in the fish embryo is also illustrated in Figure 3, with the normal embryo appearance, that is without edema in the experiment, as a comparison - the forehead and the atrium of the heart should have had a clear outline in the yolk of the egg after stage 23 for the normal embryos with a relatively small space around the heart; blurred atrium of the heart and cuvierian duct were considered as edema growth in our experiment.

Table 1: Abnormal growth in the treated groups.

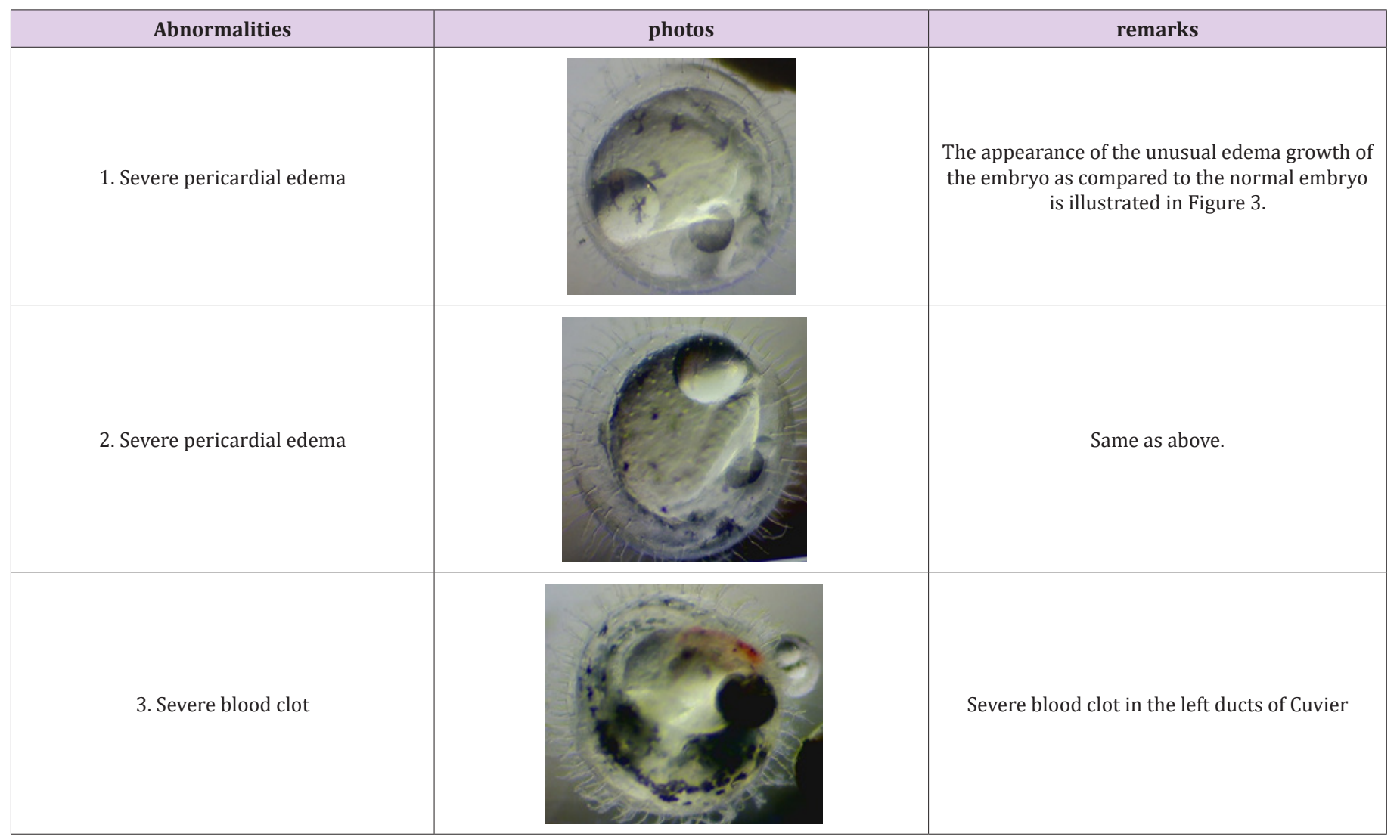




\begin{tabular}{|c|c|}
\hline 4. Small blood clots & $\begin{array}{l}\text { Small blood clots inside the developing brain } \\
\text { (possibly in the primordial midbrain channel) }\end{array}$ \\
\hline 5. Over-pigment & $\begin{array}{l}\text { Over-pigmented in the cranial region. The left } \\
\text { eye is darkened. }\end{array}$ \\
\hline 6. deformed head (tapered) \& eye cup & $\begin{array}{l}\text { The cranial roof round the forebrain appears } \\
\text { collapsed. }\end{array}$ \\
\hline Final hatched abnormal & $\begin{array}{l}\text { Abnormal fish that is unable to swim normally } \\
\text { with tingled tail. }\end{array}$ \\
\hline
\end{tabular}

Table 2: Abnormal growth in the control groups.

\begin{tabular}{|l|l|l|}
\hline Abnormalities & photos & remarks \\
\hline 1. Huge blood clot & Huge blood clot in the left duct of Cuvier, \\
possibly due to fungal infection (filamentous \\
hyphae is apparent on the surface of the \\
chorion).
\end{tabular}

The proportion of embryos with severe pericardial edema was $2.7 \%$ ( 2 out of 75 ) in the treated groups, while $0 \%$ in the controlled groups. The probability of edema in normal embryos is commonly less than $0.2 \%$. The proportion of embryos with blood clots was $2.7 \%$ ( 2 out of 75 ) in the treated groups while $1.3 \%$ (1 out of 75 ) in the controlled groups. There was also 1.3\% (1 out of 75 ) of embryos with over-pigment development and also $1.3 \%$ (1 out of 75) with deformed heads. For the final hatched fish, an abnormal fish with tingled tail was also observed in the treated groups. There was no significant difference in the average hatching days with 17.25 days and 17.14 days for the controlled and the treated groups respectively; and a difference in the hatching rate of $83.56 \%$ and $76.92 \%$ was observed between the two groups. This is summarized in Figure 4. Transcriptome analysis (by RNA sequencing) of the embryos was further carried out to investigate the underlying molecular changes for the abnormal growth at different embryonic stages. Samples from three time points were selected for transcriptome analysis in both the treated and the control groups to analyze the gene expression; they were analyzed separately for the different developmental stages of the embryos at each time point. Transcriptome analysis is an effective method to measure the expression levels of genes involved in a broad array of biological processes and signaling pathways. 


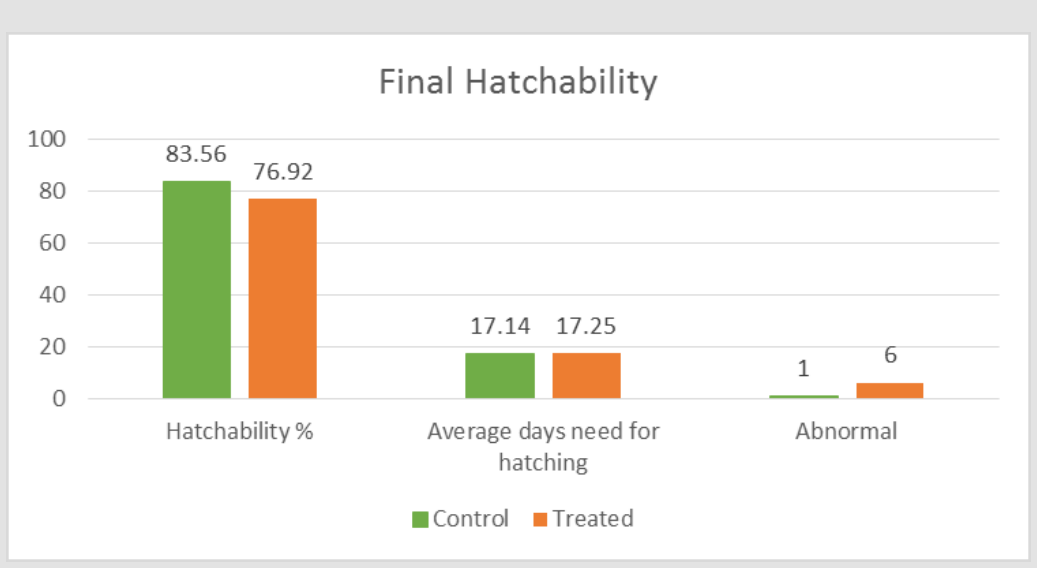

Figure 4: Histogram of the final hatchability of the repeated experiment.

The time points showing abnormal embryo growth was detected at day 3, day 5 and day 7 (which correspond to developmental stages 22 to 27), and total RNA was extracted for transcriptome analysis. Quality Checking was carried out on all the samples before RNA sequencing. The genes of the RNA samples of the treated group were compared to those of the controlled groups; those with an adjusted p-value of less than 0.05 and also with a |log2 fold change| of larger than 1 were considered as significant genes having a significant difference. In summarizing the outcomes of the analysis of the RNA sequencing 1,500 , and 30 genes were of significant gene for the 3 time points of the 3,5 , and 7 days exposures. All samples obtained from the time points of 5 and 7 days were further evaluated by functional annotation; the samples obtained from the time point of 3 days exposure were not considered for functional annotation due to only 1 significant gene was identified.

The functional annotation aims to identify any possible functions and pathways in fish embryos affected by the SMF exposure; the gene expression was first characterized to over 100 common biological processes. The outcomes of the analysis indicated three main biological processes - metabolism, cellular biosynthesis, and the organism localization process had a p-value of less than 0.05 and with over $50 \%$ of the significant genes of the two time points. The gene expression data were further characterized into molecular functions, and the outcomes of the significant molecular functions of the two time points are summarized in (Tables 3 \& 4); the protein binding and transporter activity were identified to be the main significant molecular functions. The KEGG pathway was also analyzed to identify any genomic information to the gene function; the outcomes of the KEGG pathway are shown in (Table 5). Genes related to functions of ribosome, Huntington's disease, pyrimidine metabolism, and the RNA polymerase from the treated groups were identified to be significantly different as compared to the controlled groups after a 5 day exposure; genes related to function of fat digestion and absorption for the 7 days exposure.
Table 3: The significant molecular function of 5 days exposure functional annotation (for number of genes involved $>5$ ).

\begin{tabular}{|c|c|c|c|}
\hline Term & Count & $\%$ & P-value \\
\hline G0:0005488 binding & 189 & 79.41 & 0.0062 \\
\hline GO:0005515 protein binding & 148 & 62.18 & 0.0152 \\
\hline G0:0003723 RNA binding & 34 & 14.29 & 0.0033 \\
\hline GO:0044822 $\sim$ poly(A) RNA binding & 25 & 10.50 & 0.0111 \\
\hline $\begin{array}{c}\text { GO:0005198 structural molecule } \\
\text { activity }\end{array}$ & 17 & 7.14 & 0.0303 \\
\hline $\begin{array}{c}\text { GO:0003735 structural constituent of } \\
\text { ribosome }\end{array}$ & 12 & 5.04 & $9.14 \mathrm{E}-05$ \\
\hline $\begin{array}{c}\text { GO:0016779 nucleotidyltransferase } \\
\text { activity }\end{array}$ & 7 & 2.94 & 0.0061 \\
\hline GO:0009055 electron carrier activity & 6 & 2.52 & 0.0154 \\
\hline $\begin{array}{c}\text { G0:0003899 DNA-directed RNA } \\
\text { polymerase activity }\end{array}$ & 5 & 2.10 & 0.0020 \\
\hline G0:0034062 RNA polymerase activity & 5 & 2.10 & 0.0024 \\
\hline
\end{tabular}

Table 4: The significant molecular function of 7 days exposure functional annotation (for number of genes involved $>5$ ).

\begin{tabular}{|c|c|c|c|}
\hline Term & Count & $\%$ & P-value \\
\hline $\begin{array}{c}\text { GO:0022892 substrate-specific } \\
\text { transporter activity }\end{array}$ & 8 & 47.059 & $2.46 \mathrm{E}-05$ \\
\hline G0:0005215 transporter activity & 8 & 47.059 & $6.69 \mathrm{E}-05$ \\
\hline GO:0005102 receptor binding & 6 & 35.29 & 0.0069 \\
\hline $\begin{array}{c}\text { GO:0017127 cholesterol transporter } \\
\text { activity }\end{array}$ & 5 & 29.41 & $5.48 \mathrm{E}-10$ \\
\hline $\begin{array}{c}\text { GO:0015248 sterol transporter } \\
\text { activity }\end{array}$ & 5 & 29.41 & $1.94 \mathrm{E}-09$ \\
\hline GO:0005319 lipid transporter activity & 5 & 29.41 & $2.37 \mathrm{E}-06$ \\
\hline GO:0005543 phospholipid binding & 5 & 29.41 & $2.16 \mathrm{E}-04$ \\
\hline $\begin{array}{c}\text { GO:0008289 lipid binding } \\
\text { GO:0003899 DNA-directed RNA } \\
\text { polymerase activity }\end{array}$ & 5 & 29.41 & 0.0022 \\
\hline GO:0034062 RNA polymerase activity & 5 & 2.10 & 0.0024 \\
\hline
\end{tabular}


Table 5: The significant KEGG pathway of functional annotation (for number of genes involved > 5).

\begin{tabular}{|c|c|c|c|}
\hline Category & Term & Count & P-value \\
\hline KEGG_PATHWAY - 5 days & hsa03010:Ribosome & 13 & 5.46 \\
\hline KEGG_PATHWAY - 5 days & hsa05016:Huntington's disease & 9 & 3.78 \\
\hline KEGG_PATHWAY - 5 days & hsa00240:Pyrimidine metabolism & 6 & 2.52 \\
\hline KEGG_PATHWAY - 7 days & hsa03020:RNA polymerase & 5.0033 & 2.10 \\
\hline
\end{tabular}

\section{Discussion}

This paper describes an in-vivo experimental study of the medaka fish model; it is verified that there was no significant impact on the embryo growth rate as a repeated experiment with a larger sample size; however, there is an observable difference in the numbers of abnormal growth and hatching rate between the treated and the controlled groups. A low hatching rate could be due to hatching failure, and in turn that hatching failure might be due to abnormal growth. According to the lower hatching rate and the higher abnormal growth in the treated groups as compared to the controlled groups under SMF exposure, it might be postulated that the abnormal growth could lead to the hatching failure in our experiment. As only one sample eventually hatched with malformation in the whole experiment of a comparably large sample size, it could also be postulated that the abnormal growth might not lead to any specific impact in the final formation of the embryonic bodies on hatching under SMF exposure. It is identified that in the RNA sequencing there was an obvious change in the functional annotation after a SMF exposure of 5 days - the molecular functions and the KEGG pathway related to ribosome disease, digestion and absorption of fat in the treated groups were significantly different from the control groups.

\section{Conclusion}

This paper presents an in-vivo medaka fish embryo development experiment under static magnetic field exposure with additional transcriptome analysis. It was concluded that there is no impact of SMF to the embryo growth rate, however abnormal embryo growth was observed with further functional annotation and KEGG pathway analysis. This study provides evidential data of an in-vivo experiment to supplement and clarify the ambiguity of the SMF impacts on human exposure by inference from the outcomes of the medaka fish model, for the possible health risk assessment for a human model.

\section{References}

1. Karabetsos E, Kalampaliki E, Tsanidis G, Koutounidis D, Skamnakis, et al. (2010) EMF measurements in hybrid technology cars. In Proceedings of the 6th InternationalWorkshop on Biological Effects of Electromagnetic Fields, Bodrum, Turkey.

2. Moreno Torres P, Lafoz M, Blanco M, Arribas JR (2016) Passenger exposure to magnetic fields in electric vehicles. In Modeling and Simulation for Electric Vehicle Applications; Intech: London, UK.

3. Jalilian H, Najafi K, Monazzam MR, Khosravi Y, Zamanian Z (2017) Assessment of static and extremely low frequency magnetic fields in the electric-powered trains. Int J Occup Hyg 9: 105-112.

4. Ptitsyna NG, Ponzetto A, Kopytenko YA, Ismagilov VS Korobeinikov AG (2014) Electric vehicle magnetic fields and their biological relevance. J Sci Res Rep 3: 1753-1770.

5. (2006) ICNIRP; WHO. Environmental Health Criteria 232: Static Fields; WHO: Geneva, Switzerland.

6. (1998) ICNIRP Guidelines for limiting exposure to time-varying electric, magnetic, and electro-magnetic fields (Up to $300 \mathrm{GHz}$ ). Health physics 74: 494-522.

7. (2006) Environmental Health Criteria 232: Static Fields. Geneva, World Health Organization; ISBN 92-4-157232-9.

8. Zmyślony M, Palus J, Jajte J, Dziubaltowska E, Rajkowska E (2000) DNA damage in rat lymphocytes treated in vitro with iron cations and exposed to $7 \mathrm{mT}$ magnetic fields (static or $50 \mathrm{~Hz}$ ). Mutat Res 453(1): 89-96.

9. Reina FG, Pascual LA (2001) Influence of a stationary magnetic field on water relations in lettuce seeds. Part I: theoretical considerations. Bioelectromagnetics 22: 589-595.

10. Hirai T, Nakamich N, Yoneda Y (2002) Activator protein-1 complex expressed by magnetism in cultured rat hippocampal neurons. Biochem Biophys Res Commun 292(1): 200-207.

11. Amara S, Douki T, Garel C, A Faiver, M Sakly (2009) Effects of static magnetic field exposure on antioxidative enzymes activity and DNA in rat brain. Gen Physiol Biophy 28(3): 260-265.

12. Sun W, He Y, Leung SW, Kong YC (2019) In vivo analysis of embryo development and behavioral response of medaka fish under static magnetic field exposures. Int J Environ Res Public Health 16(5): 844. 
ISSN: 2574-1241

DOI: 10.26717 /BJSTR.2020.31.005158

Weinong Sun. Biomed J Sci \& Tech Res

(C) (i) This work is licensed under Creative (c) Commons Attribution 4.0 License

Submission Link: https://biomedres.us/submit-manuscript.php

$\begin{array}{ll}\text { BIOMEDICAL } & \text { Assets of Publishing with us } \\ \text { RESEARCHES } & \text { Global archiving of articles } \\ & \text { - Immediate, unrestricted online access } \\ & \text { - Rigorous Peer Review Process } \\ \end{array}$

